\title{
Prosthetic valve endocarditis caused by Pseudomonas aeruginosa with variable antibacterial resistance profiles: a diagnostic challenge
}

Nicolas Gürtler ${ }^{1 *}$ (D), Michael Osthoff ${ }^{1}$, Florian Rueter ${ }^{2}$, Daniel Wüthrich ${ }^{3}$, Lukas Zimmerli ${ }^{4}$, Adrian Egli ${ }^{3}$ and Stefano Bassetti ${ }^{1}$

\begin{abstract}
Background: Infective endocarditis (IE) caused by gram-negative bacilli is rare. However, the incidence of this severe infection is rising because of the increasing number of persons at risk, such as patients with immunosuppression or with cardiac implantable devices and prosthetic valves. The diagnosis of IE is often difficult, particularly when microorganisms such as Pseudomonas aeruginosa, which rarely cause this infection, are involved. One of the mainstays for the diagnosis of IE are persistently positive blood cultures with the same bacteria, while polymicrobial bacteremia usually points to another cause, e.g. an abscess. The antimicrobial resistance profile of some $P$. aeruginosa strains may change, falsely suggesting an infection with several strains, thus further increasing the diagnostic difficulties.
\end{abstract}

Case presentation: A 66-year old male patient who had a transcatheter aortic valve implantation (TAVI) one year previously developed fever seven days after an elective inguinal hernia repair. During the following four weeks, P. aeruginosa with different antibiotic resistance profiles was repeatedly isolated from blood cultures. Repeated trans-esophageal echocardiograms (TEE) were negative and an infection by different $P$. aeruginosa strains was suspected. Extensive diagnostic workup for an infectious focus was performed with no results. Finally, an oscillating mass on the aortic valve was detected by TEE five weeks after the initial positive blood cultures. P. aeruginosa endocarditis was confirmed by culture of the surgically removed valve. Whole genome sequencing of the last two $P$. aeruginosa isolates (valve and blood culture) revealed identical strains, with genome mutations for AmpR, AmpD and OprD.

Conclusions: The diagnosis of prosthetic valve endocarditis is particularly difficult for several reasons. The modified Duke criteria have a lower sensitivity for patients with prosthetic valve endocarditis and the infection may be caused by "unusual" pathogens such as P. aeruginosa. Patients with repeatedly positive blood cultures should make clinicians suspicious for endocarditis even if imaging studies are negative and if isolated pathogens are "unusual". Repeatedly positive blood cultures for $P$. aeruginosa should be considered as "persistent bacteremia" (suspicious for IE) even in the presence of different antibiotic susceptibility patterns, since $P$. aeruginosa might rapidly activate or deactivate resistance mechanisms depending on antibiotic exposition.

Keywords: Endocarditis, Pseudomonas aeruginosa, Gram-negative bacilli, Transcatheter aortic valve implantation, Resistance profile, Case report

\footnotetext{
*Correspondence: nicolas.guertler2@usb.ch

${ }^{1}$ Division of Internal Medicine and Department of Clinical Research,

University Hospital Basel, University of Basel, Basel, Switzerland

Full list of author information is available at the end of the article
}

(c) The Author(s). 2019 Open Access This article is distributed under the terms of the Creative Commons Attribution 4.0 International License (http://creativecommons.org/licenses/by/4.0/), which permits unrestricted use, distribution, and reproduction in any medium, provided you give appropriate credit to the original author(s) and the source, provide a link to the Creative Commons license, and indicate if changes were made. The Creative Commons Public Domain Dedication waiver (http://creativecommons.org/publicdomain/zero/1.0/) applies to the data made available in this article, unless otherwise stated. 


\section{Background}

Infective endocarditis (IE) remains a serious disease that is still associated with significant morbidity and mortality, despite diagnostic and surgical advances [1-3]. Early stages of IE often lack distinct findings. The clinician's ability to associate miscellaneous hints, including risk factors for acquisition of IE, is a key factor for rapid diagnosis. Fever, a new murmur or worsening of a known murmur and less often $(<5 \%$ of cases) cutaneous manifestations (Janeway lesions, Osler nodes) are typical clinical signs, but patients with IE mostly present either with an unspecific "sepsis syndrome" (in the case of acute IE) or with a subacute illness without typical signs [4]. New diagnostic tools such as cardiac computed tomography (CT) scanning or 18-fluorodeoxyglucose positron emission tomography $\left({ }^{18} \mathrm{FDG}-\mathrm{PET}\right) / \mathrm{CT}$ are promising but expensive, and their precise role remains to be established [3]. The modified Duke criteria are of central importance for the evaluation of patients with suspected IE and include as major microbiological criterion "persistently positive blood cultures" [5].

$P$. aeruginosa is a very rare cause of endocarditis. In a recent Italian prospective cohort study (2004-2011) only 13 of 1722 IE episodes (0.75\%) were caused by Pseudomonas species (including two co-infections) [6], compared with 11 of 2761 IE episodes $(0.4 \%)$ in an international prospective cohort study analyzing IE cases from 2000 to 2005 [7]. However, the incidence of IE is increasing, in part because of more frequent use of cardiac implantable electronic devices and also because patients receiving transcatheter valve replacement may be at higher risk for IE $[3,4]$. In the United States, the incidence of IE increased steadily from 11 to 15 per $100^{\prime} 000$ population in the years 2000 to 2011, and the proportion of IE due to gram-negative bacteria increased from 5.3 to $8.2 \%$ [8]. Moreover, IE is currently health-care acquired in $>25 \%$ of cases [3].

For the same reasons, the epidemiology is changing also specifically for $P$. aeruginosa IE. Historically $P$. aeruginosa IE was associated with intravenous drug use [9]. However, a shift towards health care associated P. aeruginosa IE has been observed, in particular in patients with pacemaker or prosthetic valve implantation $[2,10]$. Already the large cohort study by Morpeth et al. from 2000 to 2005 showed that most non-HACEK (species other than Haemophilus species, Aggregatibacter actinomycetemcomitans, Eikenella corrodens, and Kingella species) gram-negative bacilli IE were health-care associated (57\%), while injection drug use was rare (4\%) [7]. In addition, the above-mentioned more recent Italian cohort study confirmed that a genitourinary infection focus, immunosuppressive therapy, and an indwelling cardiac implantable electronic device, but not intravenous drug use, were associated with IE caused by non-HACEK gram-negative bacilli [6].

Treatment of $P$. aeruginosa IE is difficult and complicated by biofilm formation and by the possible emergence of antibiotic resistance during treatment because of genetic polymorphisms leading for example to the increased expression of cephalosporinases, changes in efflux pump regulators, or reduced porin expression $[10,11]$. Therefore, combination antibiotic therapy is recommended and indication and timing of surgical treatment should be carefully assessed $[3,10]$.

\section{Case presentation}

A 66-year old male patient presented to the emergency department with pain in the lower abdomen and a temperature of $38.6{ }^{\circ} \mathrm{C}$. One week previously, after an incisional hernia repair, he had required a urinary catheter due to urinary retention. His past medical history was significant for a transcatheter aortic valve implantation (TAVI) due to a severe aortic stenosis 1 year earlier, and psoriasis vulgaris. On admission, the patient had a transurethral urinary catheter in place. The physical examination was normal, except for a febrile temperature and lower abdominal pain. The C-reactive protein was only mildly elevated to $16 \mathrm{mg} / \mathrm{L}$ (normal range $<10 \mathrm{mg} / \mathrm{L}$ ), and mild pyuria (10-20 leucocytes per field of view) and hematuria (5-10 erythrocytes per field of view) were present. A catheter-associated urinary tract infection was suspected. Treatment with intravenous ceftriaxone ( $2 \mathrm{~g}$ qd) was initiated and changed after 3 days to intravenous amoxicillin-clavulanate $(2.2 \mathrm{~g}$ tid). The patient continued to spike fevers up to $39.8^{\circ} \mathrm{C}$. Initial blood cultures were negative, but a repeated set of blood and urine cultures on day four was positive for $P$. aeruginosa (susceptible to all antibiotics tested, including piperacillintazobactam and ceftazidime). The antibiotic treatment was changed to intravenous piperacillin-tazobactam and later to ceftazidime. CT scans of the thorax and abdomen were unremarkable. A trans-esophageal-echocardiography (TEE) requested because of persistent fever, did not reveal any vegetation on the heart valves or other signs of infective endocarditis. Repeated blood cultures on day 15 were again positive for $P$. aeruginosa. However, now, susceptibility testing indicated resistance to piperacillintazobactam and ceftazidime. The treatment was changed accordingly to meropenem and gentamicin. Besides a mild fatigue, the patient had no localizing symptoms, and repeated TEE and abdominal and thoracic CT scans did not reveal any focus of infection. $P$. aeruginosa isolated from a blood culture on day 19 showed additional resistance to cefepime. On day 31, P. aeruginosa isolated from another blood culture changed its resistance profile one more time, now being again susceptible to piperacillintazobactam, ceftazidime and cefepime, but resistant to 
carbapenems. Antibiotic therapy was switched to cefepime and gentamicin. An ${ }^{18}$ FDG-PET/CT was not able to identify any focus of infection. After 5 weeks, the patient was transferred to a tertiary care university hospital. On day 40 , a free-floating mass $(12 \times 8 \mathrm{~mm})$ was identified on the aortic valve on TEE examination, and $P$. aeruginosa prosthetic valve endocarditis was diagnosed (Fig. 1). At this point, the patient was still febrile. Laboratory studies showed a leukocyte count of $8.9 \times 10^{9} / \mathrm{L}$ (normal range $3.5-10.0 \times 10^{9} / \mathrm{L}$ ) and a C-reactive protein of $66.2 \mathrm{mg} / \mathrm{L}$. The following day the patient successfully underwent surgical prosthetic valve replacement. The culture of the removed valve was positive for $P$. aeruginosa. Definitive antibiotic therapy consisted of intravenous cefepime, tobramycin and ciprofloxacin for additional 6 weeks (Fig. 2). The patient recovered quickly after the valve replacement and left our hospital for rehabilitation 9 days after surgery. He was doing well at the 3month follow-up.

$P$. aeruginosa isolates from blood cultures at day 31 and from the culture of the removed valve at day 41 were analyzed using whole genome sequencing (MiSeq Illumina). No genetic differences could be detected between the two isolates (cgMLST showed zero allelic differences, both strains are ST 244), providing clear evidence that the $P$. aeruginosa isolates from the blood and the aortic valve were from the same strain. We could genotypically detect mutations in the following genes: transcriptional $A m p R$ and beta-lactamase expression regulator $A m p D$, both linked to resistance against ceftazidime and piperacillin-tazobactam. In addition, we could also detect a mutation in $\operatorname{OprD}$, which is linked to carbapenem resistance. This correlates with the phenotypic findings and is in line with findings in the literature. Unfortunately, the $P$. aeruginosa isolated from the first three positive blood cultures had already been discarded and were not available for further analysis (Fig. 3).

\section{Discussion and conclusions}

We present a case of a prosthetic valve endocarditis with $P$. aeruginosa. This infection was difficult to diagnose and to treat. Initially, the presence of $P$. aeruginosa in the blood and urine after recent surgery with postoperative urinary retention suggested a surgical site infection or a urinary tract infection. P. aeruginosa accounts for $10 \%$ of healthcare-associated urinary tract infections and roughly $6 \%$ of surgical site infection in the USA [12]. Subsequently, IE was strongly suspected because of persistent bacteremia, the presence of a prosthetic valve, and lack of an alternative focus of infection (such as an abscess). Despite two TEEs and a ${ }^{18}$ FDG-PET/CT scan, it took more than a month until a final diagnosis was made. The initial isolation of $P$. aeruginosa from the urine and blood cultures (day 4) may be interpreted as catheter-associated urinary tract infection with bacteremia. Alternatively, as manifestation of endocarditis with persistent bacteremia and either secondary excretion of bacteria in the urine or concomitant urinary tract colonization. The empiric treatment with ceftriaxone may have contributed to the induction of resistance.

This case underlines the difficulty of diagnosing and treating IE caused by $P$. aeruginosa, a rare cause of IE

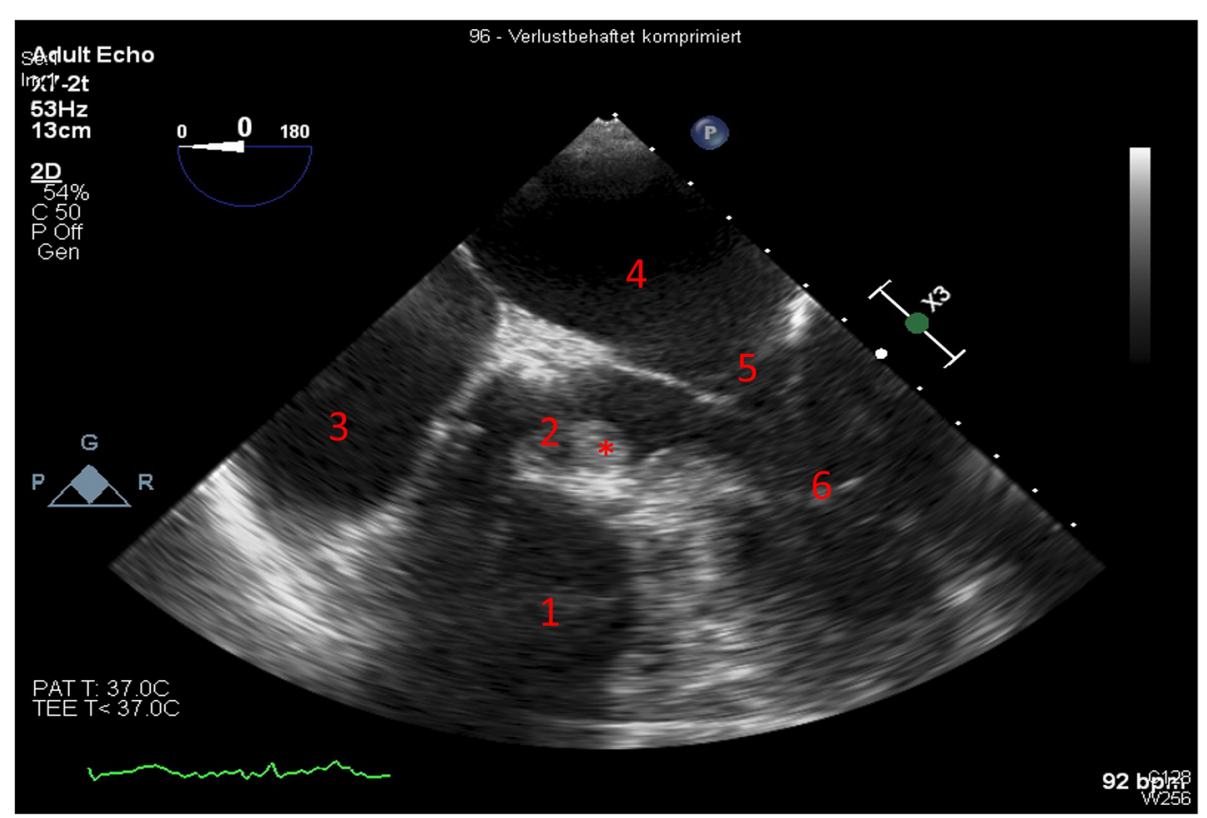

Fig. 1 TEE at day 40 with four-chamber view. * free-floating mass attached to the aortic valve. ${ }^{1}$ right ventricle, ${ }^{2}$ aortic valve, ${ }^{3}$ right atrium, ${ }^{4}$ left atrium, ${ }^{5}$ mitral valve, ${ }^{6}$ left ventricle 


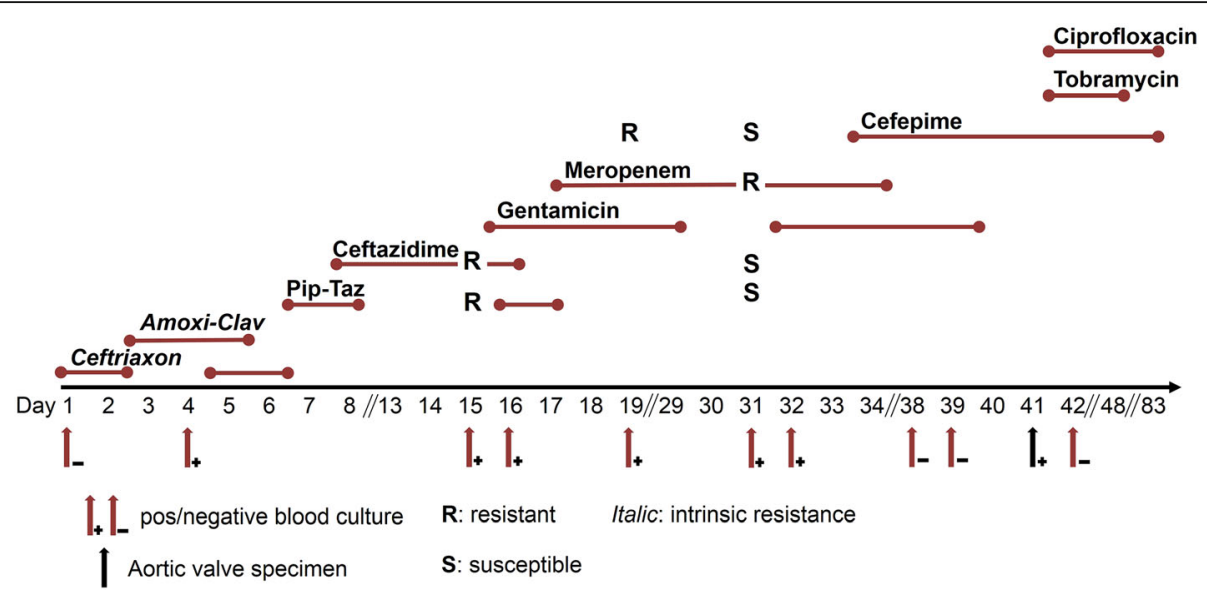

Fig. 2 Timeline of hospital stay with display of blood culture drawings (red arrow), aortic valve specimen (black arrow), antibiotic agents with duration and resistance pattern (reading example: ceftazidime was tested resistant at day 15 and tested susceptible at day 31)

and a pathogen able to form a biofilm and evade antimicrobial agents, but also able to develop resistance to multiple classes of antibiotics, even during the course of treatment $[13,14]$. P. aeruginosa can become resistant to antibiotics by the acquisition of resistance genes on plasmids or through mutations under selection pressure that modify the expression and/or function of chromosomally encoded mechanisms [13]. Carmeli et al. observed that in around $10 \%$ of patients with $P$. aeruginosa infections, new resistances developed during antibiotic treatment, and identified imipenem as a main risk factor [15]. Strains with additional unclear resistance mechanisms (e.g. possible unstable de-repression of a chromosomal AmpC $\beta$-lactamase) leading to an unstable phenotype with changing antimicrobial resistance patterns have also been described [16]. Changing phenotypes may falsely suggest the presence of multiple strains, further hindering the diagnostic process. Molecular methods, such as whole-genome-sequencing (WGS) of isolated pathogens may be helpful to understand epidemiology (e.g. outbreaks), the course of the disease (e.g. differentiating relapse of an infection by the same strain from reinfection with different strains) and to identify the mechanisms of antibiotic resistance $[11,17]$. In the presented case the development of resistance to imipenem and meropenem is most likely related to mutations of $\operatorname{OprD}$, which mediates membrane porins, and/or to an activation/upregulation of efflux pumps [18]. The appearance and disappearance of resistance to piperacillin-tazobactam, ceftazidime and cefepime may be explained by a changing production of an inducible $A m p C$ cephalosporinase (or an unstable de-repression of a chromosomal $A m p C \beta$ lactamase) [16]. As we were not able to analyze specimens of the first three positive blood cultures, it is also possible that different $P$. aeruginosa strains with variable genetic mutations were involved in the course of disease. A switch from multi-resistance to less resistance pattern in the same strain does not seem very likely.

Literature about unstable $P$. aeruginosa causing endocarditis is scarce. Lesho et al. described a similar case of

\begin{tabular}{|cccccc|}
\hline & Day 4 & Day 15 & Day 19 & Day 31 & Day 41 \\
Meropenem & $<2$ & $<2$ & $<2$ & 8 & 8 \\
Imipenem-cilastatin & 2 & 1 & 2 & $>32$ & $>32$ \\
Piperacillin-tazobactam & $<4$ & $>128$ & $>128$ & $<4$ & $<4$ \\
Ceftazidime & 2 & $>64$ & $>64$ & 0.5 & 1.5 \\
Cefepime & $<1$ & 8 & 16 & 0.75 & 2 \\
Gentamicin & $<1$ & $<1$ & $<1$ & $<1$ & $<1$ \\
Tobramycin & & & & $<1$ & $<1$ \\
Ciprofloxacin & $<0.25$ & $<0.25$ & $<0.25$ & $<0.25$ & $<0.25$ \\
Fig. 3 MICs of selected antibiotic agents, red markings for in vitro resistance & & & \\
\hline
\end{tabular}


unstable $P$. aeruginosa, in which resistance change was observed after storing $P$. aeruginosa ex vivo [16]. Contrary to our case, they observed only a one-time change of resistance pattern in the patient, whereas we described three changes over the course of the disease. However, our patient had a prolonged, persisting infection with changing selection pressures from antibiotics.

Domitrovic et al. described an infection by a $P$. aeruginosa with already broad resistance and gaining further resistance to third generation cephalosporins and piperacillin-tazobactam [11].

To our knowledge, this is the first case describing an infection with $P$. aeruginosa, which developed extensive cephalosporin, piperacillin-tazobactam and carbapenem resistance and partially lost this resistance. This highlights the ability of $P$. aeruginosa to switch on/off certain resistance mechanisms in shortest time.

A recent prospective cohort study of hospitalized patients with a cardiac device (including prosthetic heart valves) and a bacteremia showed that the risk of cardiac device-related infection is highest in patients with bacteremia due to Staphylococcus aureus, $P$. aeruginosa and Serratia marcescens [19]. In a patient with a cardiac device and bacteremia with $P$. aeruginosa one might therefore consider to start empiric therapy with a bactericidal combination of beta-lactams and aminoglycosides (preferably tobramycin), as recommended for $P$. aeruginosa endocarditis [20]. Although evidence for combination therapy in $P$. aeruginosa bacteremia is still lacking, combination therapy may also be necessary in absence of endocarditis in light of increasing resistance. Alternatively, optimized administration of beta-lactam antibiotics by continuous infusion coupled with therapeutic drug monitoring may be useful. New therapeutic approaches, as the combination of antibiotics and bacteriophages, might in the future improve the outcomes of treatment of IE caused by $P$. aeruginosa [21, 22].

In conclusion, the incidence of $P$. aeruginosa IE is increasing because of the growing number of persons at risk, such as patients with immunosuppression or with cardiac implantable devices and prosthetic valves. The diagnosis of this severe infection is often difficult and should be suspected, prompting adequate empiric antibiotic therapy, in all patients with persistent $P$. aeruginosa bacteremia, even if initial diagnostic tests are negative and particularly if implantable cardiac devices are present.

\section{Abbreviations \\ CT: Computed tomography; IE: Infective endocarditis; TAVI: Transcatheter aortic valve implantation; TEE: Trans-esophageal echocardiogram}

\section{Acknowledgements}

We thank Prof. Christine Meyer-Zürn for providing images of the transesophageal echocardiogram.
Parts of this case report have been presented at the Spring Congress 2018 of the Swiss Society of General Internal Medicine, 30.05. - 01.06.2018 (P326), Basel, Switzerland.

\section{Authors' contributions}

NG collected all clinical data and wrote the first draft of the manuscript, which was further edited by MO, AE and SB. SB, FR, LZ participated in the clinical care of the patient. AE and DW conducted the microbiology data processing and interpretation. All authors critically reviewed the manuscript for publication. All authors have read and approved the final version of this manuscript.

\section{Funding}

No funding was required for the writing of this case report.

Availability of data and materials

The data used and analyzed in this case report is available from the corresponding author on reasonable request.

\section{Ethics approval and consent to participate}

Not applicable.

\section{Consent for publication}

Written consent to publish this case report has been obtained from the patient.

\section{Competing interests}

The authors declare that they have no competing interests.

\section{Author details}

'Division of Internal Medicine and Department of Clinical Research, University Hospital Basel, University of Basel, Basel, Switzerland. ²Department of Cardiac Surgery, University Hospital Basel, University of Basel, Basel, Switzerland. ${ }^{3}$ Clinical Microbiology, University Hospital Basel, University of Basel, Basel, Switzerland. ${ }^{4}$ Department of Internal Medicine, Kantonsspital Olten, Olten, Switzerland.

Received: 27 November 2018 Accepted: 4 June 2019

Published online: 17 June 2019

\section{References}

1. Werth BJ, Carreno JJ, Reveles KR. Shifting trends in the incidence of Pseudomonas aeruginosa septicemia in hospitalized adults in the United States from 1996-2010. Am J Infect Control. 2015;43(5):465-8.

2. Amat-Santos IJ, Messika-Zeitoun D, Eltchaninoff H, Kapadia S, Lerakis S, Cheema AN, et al. Infective endocarditis after transcatheter aortic valve implantation: results from a large multicenter registry. Circulation. 2015; 131(18):1566-74.

3. Cahill TJ, Baddour LM, Habib G, Hoen B, Salaun E, Pettersson GB, et al. Challenges in infective endocarditis. J Am Coll Cardiol. 2017;69(3):325-44.

4. Wang A, Gaca JG, Chu VH. Management considerations in infective endocarditis: a review. JAMA. 2018;320(1):72-83.

5. Baddour LM, Wilson WR, Bayer AS, Fowler VG Jr, Tleyjeh IM, Rybak MJ, et al. Infective endocarditis in adults: diagnosis, antimicrobial therapy, and Management of Complications: a scientific statement for healthcare professionals from the American Heart Association. Circulation. 2015; 132(15):1435-86.

6. Falcone M, Tiseo G, Durante-Mangoni E, Ravasio V, Barbaro F, Ursi MP, et al. Risk factors and outcomes of endocarditis due to non-HACEK gram-negative bacilli: data from the prospective multicenter Italian endocarditis study cohort. Antimicrob Agents Chemother. 2018;62(4).

7. Morpeth S, Murdoch D, Cabell CH, Karchmer AW, Pappas P, Levine D, et al. Non-HACEK gram-negative bacillus endocarditis. Ann Intern Med. 2007; 147(12):829-35.

8. Pant S, Patel NJ, Deshmukh A, Golwala H, Patel N, Badheka A, et al. Trends in infective endocarditis incidence, microbiology, and valve replacement in the United States from 2000 to 2011. J Am Coll Cardiol. 2015;65(19):2070-6.

9. Wieland M, Lederman MM, Kline-King C, Keys TF, Lerner PI, Bass SN, et al. Left-sided endocarditis due to Pseudomonas aeruginosa. A report of 10 cases and review of the literature. Medicine (Baltimore). 1986;65(3):180-9. 
10. Hagiya H, Tanaka T, Takimoto K, Yoshida H, Yamamoto N, Akeda Y, et al. Non-nosocomial healthcare-associated left-sided Pseudomonas aeruginosa endocarditis: a case report and literature review. BMC Infect Dis. 2016;16(1):431.

11. Domitrovic TN, Hujer AM, Perez F, Marshall SH, Hujer KM, Woc-Colburn LE, et al. Multidrug resistant Pseudomonas aeruginosa causing prosthetic valve endocarditis: a genetic-based Chronicle of Evolving Antibiotic Resistance. Open Forum Infect Dis. 2016;3(4):ofw188.

12. Weiner $L M$, Webb AK, Limbago B, Dudeck MA, Patel J, Kallen AJ, et al. Antimicrobial-resistant pathogens associated with healthcare-associated infections: summary of data reported to the National Healthcare Safety Network at the Centers for Disease Control and Prevention, 2011-2014. Infect Control Hosp Epidemiol. 2016;37(11):1288-301.

13. Lister PD, Wolter DJ, Hanson ND. Antibacterial-resistant Pseudomonas aeruginosa: clinical impact and complex regulation of chromosomally encoded resistance mechanisms. Clin Microbiol Rev. 2009:22(4):582-610.

14. Mulcahy $L R$, Isabella VM, Lewis $K$. Pseudomonas aeruginosa biofilms in disease. Microb Ecol. 2014;68(1):1-12.

15. Carmeli Y, Troillet N, Eliopoulos GM, Samore MH. Emergence of antibioticresistant Pseudomonas aeruginosa: comparison of risks associated with different antipseudomonal agents. Antimicrob Agents Chemother. 1999; 43(6):1379-82.

16. Lesho E, Snesrud E, Kwak Y, Ong A, Maybank R, Laguio-Vila M, et al. Pseudomonas endocarditis with an unstable phenotype: the challenges of isolate characterization and Carbapenem stewardship with a partial review of the literature. Antimicrob Resist Infect Control. 2017;6:87.

17. Roach DJ, Burton JN, Lee C, Stackhouse B, Butler-Wu SM, Cookson BT, et al. A year of infection in the intensive care unit: prospective whole genome sequencing of bacterial clinical isolates reveals cryptic transmissions and novel microbiota. PLoS Genet. 2015;11(7):e1005413.

18. Poole K. Multidrug efflux pumps and antimicrobial resistance in Pseudomonas aeruginosa and related organisms. J Mol Microbiol Biotechnol. 2001;3(2):255-64.

19. Maskarinec SA, Thaden JT, Cyr DD, Ruffin F, Souli M, Fowler VG. The risk of cardiac device-related infection in Bacteremic patients is species specific: results of a 12-year prospective cohort. Open Forum Infect Dis. 2017;4(3): of 132 .

20. Habib G, Lancellotti P, Antunes MJ, Bongiorni MG, Casalta JP, Del Zotti F, et al. 2015 ESC guidelines for the management of infective endocarditis: the task force for the Management of Infective Endocarditis of the European Society of Cardiology (ESC). Endorsed by: European Association for CardioThoracic Surgery (EACTS), the European Association of Nuclear Medicine (EANM). Eur Heart J. 2015;36(44):3075-128.

21. Pires DP, Vilas Boas D, Sillankorva S, Azeredo J. Phage therapy: a step forward in the treatment of Pseudomonas aeruginosa infections. J Virol. 2015;89(15):7449-56.

22. Oechslin F, Piccardi P, Mancini S, Gabard J, Moreillon P, Entenza JM, et al. Synergistic interaction between phage therapy and antibiotics clears Pseudomonas aeruginosa infection in endocarditis and reduces virulence. J Infect Dis. 2017:215(5):703-12

\section{Publisher's Note}

Springer Nature remains neutral with regard to jurisdictional claims in published maps and institutional affiliations.

Ready to submit your research? Choose BMC and benefit from:
- fast, convenient online submission
- thorough peer review by experienced researchers in your field
- rapid publication on acceptance
- support for research data, including large and complex data types
- gold Open Access which fosters wider collaboration and increased citations
- maximum visibility for your research: over 100M website views per year
At BMC, research is always in progress.
Learn more biomedcentral.com/submissions

